\title{
VIDROS AUTOMOBILÍSTICOS COMO VESTÍGIOS DE CENA DE CRIME: UMA ABORDAGEM MULTIVARIADA
}

\author{
Caio H. P. Rodrigues ${ }^{\mathrm{a}, \mathrm{b}}$ e Aline T. Bruni ${ }^{\mathrm{a}, \mathrm{b}, *,(1)}$
}

aDepartamento de Química, Faculdade de Filosofia, Ciências e Letras de Ribeirão Preto, Universidade de São Paulo, 14040-030 Ribeirão Preto - SP, Brasil

'bINCT-Forense, Departamento de Química, Faculdade de Filosofia, Ciências e Letras de Ribeirão Preto, Universidade de São Paulo, 14040-030 Ribeirão Preto - SP, Brasil

Recebido em 19/09/2020; aceito em 05/01/2021; publicado na web em 18/02/2021

\begin{abstract}
AUTOMOBILISTIC GLASSES AS CRIME SCENE TRACES: A MULTIVARIATE APPROACH. Glasses are common trace evidence elements in crime scenes, and the analysis of this material can be essential for evaluating different criminal dynamics. This work aimed to analyze the possibility of differentiating and classifying windshield glass using multivariate analysis methods. Automotive glass fragments from different vehicle brands were evaluated according to internal and external faces. We have collected from literature EDXRF (Energy Dispersive X-ray Fluorescence) data for different oxides concentrations. These data were organized in a matrix with 56 samples and nine variables. We applied unsupervised (PCA, Principal Component Analysis) and supervised (SIMCA, Soft Interclass Modeling Classification Analogy) methods. We assessed the classification responses through ROC (Receiver Operating Characteristics). As a result, the PCA indicated the presence of two groups of glasses in three main components. SIMCA verified the unsupervised classification, and the distances and interclass residues parameters were adequate with no outliers. The ROC analysis indicated a sensitivity of 0.793 , a specificity of 0.815 , and an efficiency of 0.804 for predictions. We concluded that multivariate analysis was successful in discriminating between the internal and external faces of automotive glasses.
\end{abstract}

Keywords: glass; multivariate analysis; forensic sciences; crime scene.

\section{INTRODUÇÃO}

Existe uma grande casuística envolvendo acidentes de trânsito. De acordo com o relatório do Ministério da Infraestrutura, ocorrem cerca de oito acidentes de trânsito por hora no Brasil. Em valores absolutos ocorreram aproximadamente 69 mil ocorrências e dessas, 5,3 mil com vítimas, de acordo com o Anuário Estatístico de Segurança Rodoviária de 2020. ${ }^{1}$ Há muitos acidentes nos quais as mortes poderiam ser evitadas caso as vítimas fossem adequadamente socorridas. A omissão de socorro é penalmente punível de acordo com o Art. 304 do Código de Trânsito Brasileiro. ${ }^{2}$

Dentro das ciências forenses o local no qual se desenrolaram os fatos da dinâmica criminosa é chamado de local de crime. De forma genérica, esses espaços podem ser entendidos como o ambiente físico e/ou digital em que um crime ocorreu. ${ }^{3}$ Toda vez que uma ação criminosa ocorre, deve haver uma investigação na qual o local de crime é o ponto de partida para a coleta de vestígios. ${ }^{4}$

Para casos envolvendo eventos relacionados a trânsito os locais de crime podem ser espaços extremamente complexos. ${ }^{5}$ Isso se deve a uma ampla variedade de tipos ou fragmentos de materiais que podem ser encontrados ${ }^{6}$ e identificados como vestígios no decorrer da fase de inquérito policial.

A coleta desses materiais deve seguir as etapas da cadeia de custódia previstas no Art. 158-B do Código de Processo Penal Brasileiro. ${ }^{7}$ Dentre esses passos, os exames forenses destacam-se na etapa de processamento do código (Art. 158-B - VIII), na qual estão a identificação, a classificação e a comparação de objetos. ${ }^{4}$ De uma maneira geral, o exame de vestígios pode ser realizado no local do crime (in situ) e também em laboratórios com equipamentos adequados (in lab). A avaliação de vestígios recolhidos da cena do

*e-mail: aline.bruni@usp.br crime fundamenta-se no interesse de descobrir a dinâmica delituosa, a autoria e se esses materiais estão relacionados ao fato. ${ }^{6,8}$

Existem vários possíveis indicadores presentes no local do crime que a perícia pode usar para tentar estabelecer a materialidade do delito. Há trabalhos sobre análise de DNA, ${ }^{9-11}$ estimativa de velocidade, ${ }^{12-15}$ uso de substâncias lícitas ou ilícitas, ${ }^{16-19}$ medicina legal, ${ }^{20,21}$ inteligência forense, ${ }^{22-24}$ marcas de frenagem,,${ }^{25-27}$ lâmpadas, ${ }^{28,29}$ impacto em vidros, ${ }^{30,31}$ amostras de tinta, propostas de novas metodologias ${ }^{32-34}$ dentre outros, como os microvestígios. ${ }^{35,36}$ Esses materiais podem ter variações em suas dimensões, desde uma escala macroscópica até pequenos fragmentos ou materiais biológicos. ${ }^{37}$ Dentre os possíveis microvestígios encontrados em um local de crime existem os vidros.

As análises periciais de materiais vítreos, classicamente, utilizavam avaliação das propriedades físicas, como densidade, dureza e índice de refração. ${ }^{38,39}$ Esses métodos foram utilizados pelo fato de serem técnicas não destrutivas ou pouco destrutivas, $o$ que é importante para que os vestígios possam ser preservados. ${ }^{40}$ Entretanto, essas características, conseguem excluir possibilidades de fragmentos, mas não podem relacioná-los a sua origem, ${ }^{41} \operatorname{logo}$, em um contexto forense essas técnicas podem não conseguir diferenciar os vestígios. ${ }^{42}$

Dessa forma, com o aperfeiçoamento dos métodos analíticos de preparo de amostras e melhora na sensibilidade dos equipamentos de análise, outras técnicas ganharam destaque ${ }^{43,44}$ nas avaliações forenses. ${ }^{45}$

\section{Os vidros como vestígios forenses}

Segundo a ASTM (American Society for Testing and Materials) ${ }^{46}$ vidro é um produto inorgânico de fusão que foi esfriado até uma condição rígida sem cristalização ou, ainda, pode-se entender que vidro é um líquido super-resfriado, de estrutura amorfa que não apresenta uma rede cristalina regular. ${ }^{47,48}$ 
Existem diversos tipos de vidros, os mais comuns são os tipos silicatos. Esses são fundamentalmente constituídos de um átomo de oxigênio $(\mathrm{O})$ por dois átomos de silício $(\mathrm{Si})$ formando uma estrutura tetraédrica. ${ }^{46,49}$ Entretanto, a continuidade dessa rede pode ser interrompida para conferir diferentes propriedades,${ }^{50}$ pois suas características estruturais dependem da composição química. ${ }^{51}$ Essas alterações nas propriedades são feitas com a adição de óxidos à mistura. ${ }^{52}$ Caso fosse adicionado óxido de sódio, por exemplo, o vidro resultante teria maior fluidez e expansibilidade, porém seria mais frágil do que o vidro sem esse óxido. Por outro lado, se fosse acrescentado óxido de alumínio, a durabilidade aumentaria. Com o óxido de chumbo haveria um aumento da densidade. ${ }^{49,53}$ Além das propriedades físicas, com a adição dos óxidos de fósforo, itérbio e elementos terras raras podem-se alterar propriedades ópticas. ${ }^{54-56}$

Dessa forma, ter informações sobre a composição dos vidros torna-se importante não somente para o controle de qualidade e conferência de propriedades específicas, mas também nas ciências forenses. É possível, por exemplo, relacionar a composição dos vidros à sua geolocalização de produção, como já relatado para solos. ${ }^{57-61}$

Seguindo as boas práticas laboratoriais, ${ }^{43}$ dentre as diversas técnicas analíticas que podem ser utilizadas nas análises dessas amostras, destacam-se as espectroanalíticas, como: a espectrometria de absorção atômica (AAS - Atomic Absorption Spectrometry), ${ }^{62-64}$ a Microscopia Eletrônica de Varredura com uma espectrometria de raios- X de energia dispersiva (SEM-EDX - Energy Dispersive X-Ray Spectroscopy with Scanning Electron Microscopy), $, 65,66$ a Espectrometria de emissão atômica por Plasma acoplado indutivamente (ICP-AES - Inductively Coupled Plasma - Atomic Emission Spectrometry), ${ }^{37,52,67,68}$ a espectrometria de emissão de raios-X por partícula induzida (PIXE - Particle-induced X-ray emission), ${ }^{69}$ a espectrometria de fluorescência de raios-X de energia dispersiva (ED-XRF - Energy Dispersive X-ray Fluorescence) ${ }^{70-72}$ IR (Infrared spectroscopy), ${ }^{73,74}$ e LIBS (Laser Induced Breakdown Spectroscopy). ${ }^{75-80}$ Podemos, ainda, citar as análises por isótopos estáveis. ${ }^{81-83}$

Devido ao desenvolvimento desses e outros métodos de análise, as características específicas de cada tipo de vidro devem respeitar um controle de qualidade, como as NBR (Norma Técnica) 9491:2015, ${ }^{84}$ 9492:2014, $,^{85} 7199: 2016 .{ }^{86}$ Esse processo torna-se necessário, pois há uma infinidade de possibilidades para a criação de diversas composições de vidros. Assim, os vidros e as análises diretas de sólidos compõem amostras de complexa análise tanto no âmbito do interesse forense quanto em outros possíveis. ${ }^{43}$

Há ainda aspectos importantes a serem observados no preparo de amostras, ${ }^{43,87}$ como o possível aumento de erros sistemáticos devido às contaminações (asfalto, tintas, solo, poluição dentre outros), ${ }^{88}$ efeitos de matriz (autoabsorção e/ou espessura), ${ }^{89}$ ou a perda de analitos por volatilização. Quando se leva a escala de concentrações a traços ou ultra traços ${ }^{90}$ ou tenta-se analisar todo o volume de dados ${ }^{44,91-93}$ sem uma abordagem adequada, as dificuldades tornamse ainda maiores.

Quando avalia-se esse conjunto de aspectos em analitos complexos, o que geralmente compreende muitas variáveis (sinais químicos) e muitas amostras, a interpretação dessas respostas e até mesmo as análises pode ser uma tarefa não trivial. ${ }^{94}$ Além desses fatores, quando o foco são os exames forenses, um desafio adicional de análise surge.$^{95}$ Como esses materiais são vestígios comumente encontrados em eventos relacionados a trânsito, ${ }^{39}$ o correto referenciamento dos diferentes fragmentos de vidros presentes no local do crime é crucial. Os mecanismos de identificação e análise tornam-se muito importantes para auxiliar na reconstrução da dinâmica dos fatos e na determinação da materialidade da ação. ${ }^{96}$
Os métodos quimiométricos têm se mostrado de grande utilidade para auxiliar a avaliação conjunta de muitas respostas químicas de amostras sob investigação. ${ }^{97-100}$ De forma mais específica, no contexto forense, a quimiometria auxilia no confronto e avaliação de materiais por reconhecimento de padrões..$^{91,94}$

Dentre os métodos que processam esse reconhecimento de padrões, temos os exploratórios, como PCA (Principal Component Analysis), ${ }^{100}$ e os classificatórios, como o método SIMCA (Soft Independent Modeling Class Analogy). ${ }^{101}$ Com a PCA, avalia-se a organização natural das informações, sem nenhuma indicação prévia sobre grupos ou classes. ${ }^{102-105}$ No método SIMCA, por outro lado, é necessário indicar previamente os grupos ou classes, para que esses possam ser utilizados para criar modelos preditivos de classificação para outras amostras. ${ }^{106-110}$ Logo, a utilização desses e outros métodos quimiométricos para os diferentes tipos de vidro, com diferentes procedências, tem sido explorada. ${ }^{111-113}$

Dessa forma, o presente trabalho tem como objetivo analisar as possibilidades de distinção por PCA e criação de um modelo classificatório empregando SIMCA entre amostras de vidros disponíveis na literatura. ${ }^{70}$ Essa discussão fomentará bases para emprego de metodologias combinadas para análise de vidro como microvestígio no contexto forense e em específico para este trabalho, no contexto de crimes de trânsito.

\section{PARTE EXPERIMENTAL}

Para as análises quimiométricas, foram utilizados dados disponíveis na literatura de Civici e Vataj. ${ }^{70}$ As amostras são referentes a 28 fragmentos de vidros automotivos (para-brisas) de 26 modelos de veículos. Esses fragmentos foram analisados utilizando a técnica EDXRF (Energy Dispersive X-ray Fluorescence) em um sistema de excitação de alvo secundário (cobre - $\mathrm{Cu}$ e molibdênio - Mo) para elementos de baixo e médio número atômico. As análises foram conduzidas nas duas chapas vítreas que compõe o para-brisas.

A matriz de dados foi organizada com os fragmentos dos vidros dos diferentes veículos sendo 56 amostras (28 para o Lado Interno e 28 para o Lado Externo conforme indicado na Figura 1) e 9 variáveis sendo as concentrações dos diferentes óxidos analisados (Tabela 1S disponível no Material Suplementar). Esses foram: sódio $\left(\mathrm{Na}_{2} \mathrm{O}\right)$, magnésio $(\mathrm{MgO})$, manganês $(\mathrm{MnO})$, alumínio $\left(\mathrm{Al}_{2} \mathrm{O}_{3}\right)$, silício $\left(\mathrm{SiO}_{2}\right)$, potássio $\left(\mathrm{K}_{2} \mathrm{O}\right)$, cálcio $(\mathrm{CaO})$, titânio $\left(\mathrm{TiO}_{2}\right)$ e ferro $\left(\mathrm{Fe}_{2} \mathrm{O}_{3}\right)$.

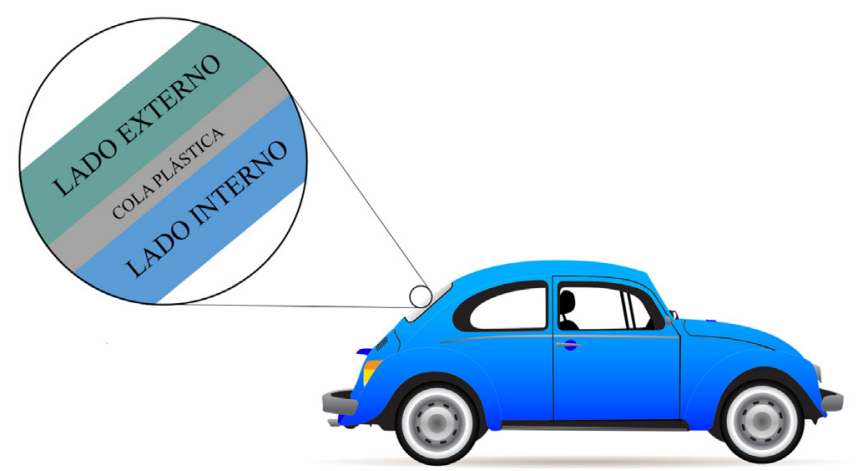

Figura 1. Representação esquemática dos lados avaliados em perspectiva com o veículo

A análise dos dados foi conduzida utilizando duas abordagens multivariada, sendo: i) uma não supervisionada e exploratória, a PCA; e ii) uma abordagem supervisionada e classificatória, o método SIMCA. A PCA explora as características dos valores introduzidos, promovendo a redução da dimensionalidade do sistema, organizando as amostras de acordo com as similaridades naturais. ${ }^{114}$ 
A SIMCA, nesse caso, foi utilizada para auxiliar a verificar se a classificação exploratória feita previamente pode embasar um modelo supervisionado, sendo possível mensurar os erros de classificação. ${ }^{91,108}$ Como pré-tratamento, os dados foram centrados na média para eliminar o erro aleatório, uma vez que foram medidos a partir da mesma técnica. ${ }^{97}$ As análises foram desenvolvidas no programa Pirouette v.4.5 (Infometrix). ${ }^{115}$

Como forma de avaliar as respostas da classificação realizadas por SIMCA, utilizou-se a análise ROC (Receiver Operating Characteristics). Essa abordagem possibilita avaliar a sensibilidade (taxa de positivos verdadeiros) e especificidade (taxa de negativos verdadeiros). Comumente, a análise ROC relaciona-se a respostas médicas, porém, tem sido utilizada para outras áreas como a química. ${ }^{97,116,117}$ Para essa avaliação foi utilizada a tabela de contingência 2x2 (Tabela 1). A codificação indicada nas Tabelas $1 \mathrm{e}$ 2 foram verdadeiro-positivo ( $\mathrm{TP}$ - true-positive), falso-positivo (FP - false positive), falso-negativo ( $\mathrm{FN}$ - false-negative) e verdadeironegativo ( $\mathrm{TN}$ - true negative). As equações utilizadas para obtenção das respostas foram agrupadas na Tabela $2 .{ }^{118-120}$

Tabela 1. Tabela codificada de contingenciamento $2 \times 2^{118-120}$

\begin{tabular}{ccc}
\hline \multirow{2}{*}{ Indicação } & \multicolumn{2}{c}{ Predição } \\
\cline { 2 - 3 } & Lado Interno & Lado Externo \\
\hline Lado Interno & $\mathbf{T P}$ & $\mathbf{F P}$ \\
Lado Externo & $\mathbf{F N}$ & $\mathbf{T N}$ \\
Total de amostras analisadas & $\mathbf{T P}+\mathbf{F N}$ & $\mathbf{F P}+\mathbf{T N}$ \\
\hline
\end{tabular}

Tabela 2. Descrição dos parâmetros de performance ${ }^{118-120}$

\begin{tabular}{cc}
\hline Sensibilidade & TP \\
\hline $\begin{array}{c}\text { Taxa de falsos negativos }(\mathrm{FN}) \\
(1-\text { sensibilidade })\end{array}$ & $\frac{\mathbf{T P N}+\mathbf{F N}}{\mathbf{T P}+\mathbf{F N}}$ \\
\hline $\begin{array}{c}\text { Especificidade } \\
\text { Taxas de falsos positivos (FP) } \\
(1-\text { especificidade })\end{array}$ & $\frac{\mathbf{T N}}{\mathbf{T N}+\mathbf{F P}}$ \\
\hline Acurácia/Eficiência & $\frac{\mathbf{F P}}{\mathbf{T N}+\mathbf{F P}}$ \\
\hline
\end{tabular}

As respostas variam de 0 a $1 .{ }^{121} \mathrm{~A}$ sensibilidade indicada no teste pode ser entendida como a capacidade do modelo em identificar uma amostra que realmente pertencente ao Lado Interno do para-brisas. Quanto maior o seu valor menor será a taxa de falsos negativos. A especificidade pode ser entendida como a probabilidade do modelo em identificar uma amostra classificada errada, assim, quanto maior o seu valor menor a taxa de falsos positivos. ${ }^{122}$ Esquematicamente, o procedimento realizado foi resumido na Figura 2.

\section{RESULTADOS E DISCUSSÕES}

No caso avaliado, os vidros foram analisados utilizando EDXRF (Energy Dispersive X-Ray Fluorescence).$^{70}$ Essa técnica permite a detecção de elementos com números atômicos entre o sódio $(\mathrm{Na})$ e o urânio (U). O limite de detecção dessa técnica é da ordem de $\mathrm{mg} \mathrm{kg}^{-1} \cdot{ }^{90}$ Devido a essa possibilidade de análise em nível traço, a abordagem se mostra adequada para as amostras analisadas, principalmente sob a ótica forense.

Inicialmente, realizamos uma seleção de variáveis com o intuito de remover as respostas que apresentavam maiores concentrações dos óxidos e que são os constituintes básicos dos vidros tipo sodocálcico. ${ }^{124,125}$ Dessa forma, removemos os dados para os óxidos de silício $\left(\mathrm{SiO}_{2}\right)$, cálcio $(\mathrm{CaO})$ e sódio $\left(\mathrm{Na}_{2} \mathrm{O}\right)$, pois são os constituintes majoritários dos vidros e suas concentrações apresentam pouca variação nas amostras. Assim, avaliamos os óxidos de magnésio $(\mathrm{MgO})$, manganês $(\mathrm{MnO})$, alumínio $\left(\mathrm{Al}_{2} \mathrm{O}_{3}\right)$, potássio $\left(\mathrm{K}_{2} \mathrm{O}\right)$, titânio $\left(\mathrm{TiO}_{2}\right)$ e ferro $\left(\mathrm{Fe}_{2} \mathrm{O}_{3}\right)$.

Como os dados foram obtidos dos Lados Interno e Externo, isto é, foram avaliadas as duas placas vítreas que compunham os para-brisas dos veículos, realizamos uma PCA para analisarmos as respostas dos dados e quais variáveis seriam as responsáveis pela organização natural das amostras. Para isso, utilizamos como pré-tratamento os dados centrados na média, pois os valores são da mesma fonte.

Nesse conjunto de dados, ${ }^{70}$ removemos os constituintes majoritários, uma vez que eles são encontrados em grandes quantidades em todas as amostras e não oferecem informações que fossem discriminatórias. Desse modo, a PCA fragmenta em duas respostas as informações originais, as matrizes dos Escores e dos Pesos. A primeira refere-se às amostras reescritas no novo sistema de eixos (novo espaço amostral redimensionado), que são as componentes principais ( $\mathrm{PC}$ - Principal Component). Os Pesos representam a distribuição das variáveis pelas novas componentes principais e como elas influenciam na organização as amostras.

Na Figura 3 estão os Escores obtidos com a PCA. Em vermelho destacam-se as amostras pertencentes ao Lado Interno e, em preto, as do Lado Externo.

Com a PCA, 98,80\% de toda a informação inicial do sistema foram reescritas e estão contidas nas três primeiras componentes principais. A Tabela 3 resume a porcentagem acumulada em cada uma das componentes principais.

Os Pesos foram sistematizados na Tabela 4, onde pode-se observar a influência de cada uma das variáveis no arranjo das amostras nas componentes principais.

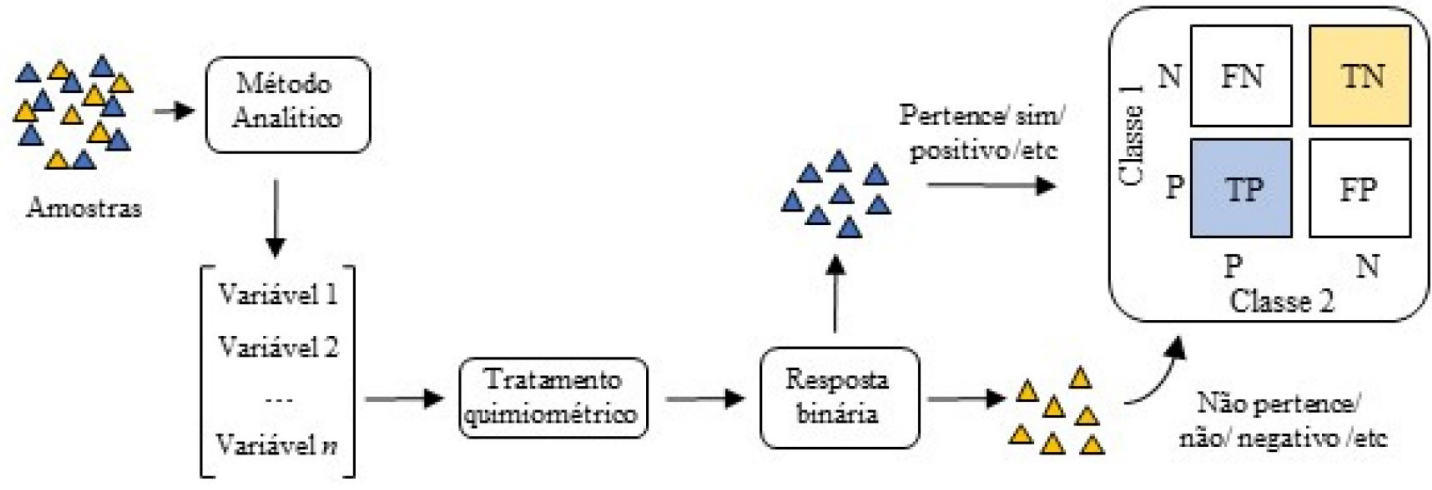

Figura 2. Representação esquemática do procedimento quimiométrico efetuado para a classificação das amostras (Adaptado de López ${ }^{118}$ e Nachtigall ${ }^{123}$ ) 


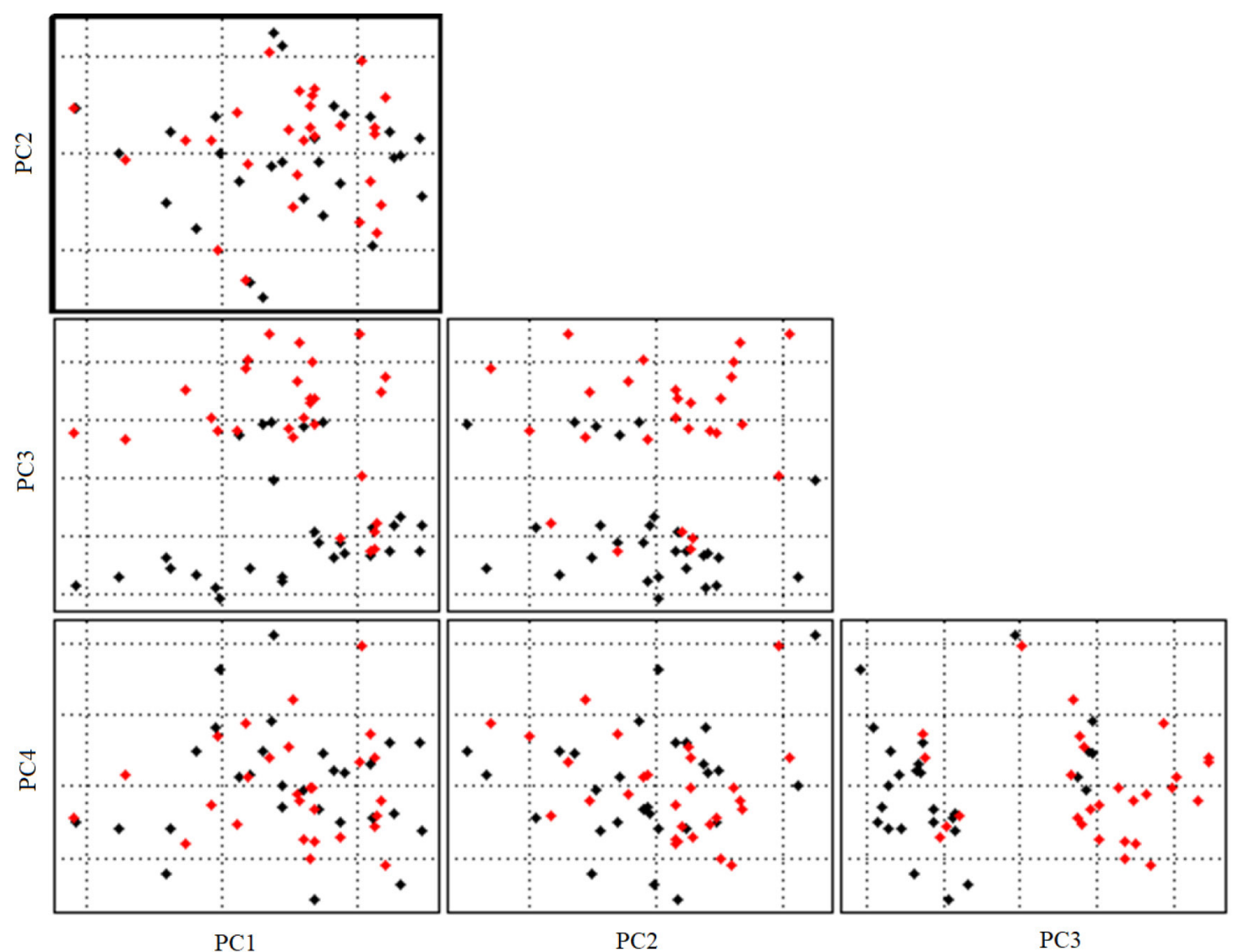

Figura 3. Resultado da PCA (Escores) para uma avaliação exploratória não supervisionada das diferentes composições das amostras

Tabela 3. Relação entre as componentes principais e o acúmulo de informação

\begin{tabular}{lc}
\hline & \% Acúmulo de informação \\
\hline PC1 & 76,55 \\
PC2 & 94,96 \\
PC3 & 98,80 \\
PC4 & 99,96 \\
PC5 & 99,99 \\
PC6 & 100,00 \\
\hline
\end{tabular}

Na Figura 3 observamos a formação de dois grupos, onde a separação entre eles ocorre na terceira componente principal. Ao avaliar-se a Tabela 4, os valores dos pesos, compreende-se que, fundamentalmente, a separação ocorre entre os óxidos de ferro e potássio. O primeiro está presente na composição do Lado Externo, utilizado para bloquear parte da luminosidade e possui tonalidade verde observada em alguns para-brisas. ${ }^{126} \mathrm{O}$ óxido de potássio foi o que apresentou maior peso para agrupar as amostras do Lado Interno, o que pode indicar a característica da matéria prima utilizada. ${ }^{57-61}$

A diferenciação entre esses vidros se torna alvo de pesquisa, pois estruturalmente esses para-brisas são constituídos de duas placas vítreas. Essas são unidas por um polímero que confere mais resistência ao choque e, em caso de quebra, mais segurança. ${ }^{70}$ Além disso, com essa indicação das composições, podem-se utilizar essas informações como ferramenta para a elucidação da dinâmica da ação.

Desse modo, para avaliarmos os agrupamentos observados previamente pela PCA e se eles formam grupos que possam ser utilizados para diferenciar outras amostras, utilizou-se a análise supervisionada, SIMCA. O agrupamento observado pela PCA na avaliação exploratória serviu de entrada para a modelagem dos dados por SIMCA. A proposta foi gerar um sistema classificatório que possa ser aplicado para prever futuras amostras analisadas por EDXRF em um contexto forense. Nesse caso, pode-se identificar se os fragmentos são da parte externa ou interna de um veículo. Essa resposta pode auxiliar na descoberta de diversos fatores envolvendo a dinâmica do choque em caso de perícias de trânsito, uma vez que com fragmentos de diferentes origens de um mesmo veículo, ou de veículos diferentes, podem-se fazer inferências sobre a posição relativa em que estava a vítima. Por outro lado, com os dados discutidos neste trabalho, não é possível fazer inferências mais específicas sobre as marcas dos veículos.

Tabela 4. Valores numéricos dos matriz Pesos da PCA

\begin{tabular}{|c|c|c|c|c|c|c|}
\hline & $\mathrm{PC} 1$ & PC2 & PC3 & PC4 & PC5 & PC6 \\
\hline $\mathrm{MgO}$ & 0.818 & -0.568 & 0.017 & 0.090 & 0.004 & -0.001 \\
\hline $\mathrm{Al}_{2} \mathrm{O}_{3}$ & 0.574 & 0.810 & 0.043 & -0.109 & -0.001 & 0.001 \\
\hline $\mathrm{K}_{2} \mathrm{O}$ & 0.000 & 0.142 & -0.292 & 0.946 & 0.015 & -0.011 \\
\hline $\mathrm{TiO}_{2}$ & -0.003 & 0.001 & -0.003 & -0.018 & 0.999 & -0.045 \\
\hline $\mathrm{MnO}$ & 0.000 & 0.001 & 0.006 & 0.013 & 0.046 & 0.999 \\
\hline $\mathrm{Fe}_{2} \mathrm{O}_{3}$ & -0.040 & 0.017 & 0.955 & 0.292 & 0.008 & -0.009 \\
\hline
\end{tabular}


Desse modo, a Tabela 5 reúne as informações obtidas para o modelo classificatório obtido por SIMCA para cada uma das chapas vítreas. Para a análise dos resultados, temos três indicadores: classificações erradas (misclassifications), resíduos interclasses e distâncias interclasses. No primeiro caso, observamos as classificações (Lado Interno, Lado Externo e não classificação) das amostras perante o modelo elaborado. Os valores dos resíduos interclasses deverão ser maiores nas classes diferentes do que na classe utilizada como comparação. Por fim, os valores das distâncias interclasses deverão ser maiores entre as classes diferentes.

Tabela 5. Resultado da análise realizada por SIMCA

\begin{tabular}{lccc}
\hline Misclassification & & & \\
\hline & $\begin{array}{c}\text { Classe 1 } \\
\text { (Predição, } \\
\text { 2PC) }\end{array}$ & $\begin{array}{c}\text { Classe 2 } \\
\text { (Predição, } \\
\text { 2PC) }\end{array}$ & $\begin{array}{c}\text { Sem } \\
\text { classificação }\end{array}$ \\
\hline Classe 1 (original) & 23 & 5 & 0 \\
Classe 2 (original) & 6 & 22 & 0 \\
\hline Resíduos Interclasses & & & \\
\hline & Classe 1 & Classe 2 \\
\hline Classe 1 & 0.1219 & 0.2306 \\
Class 2 & 0.2377 & & 0.1301 \\
\hline Distâncias Interclasses & & & Classe 2 \\
\hline \multicolumn{3}{c}{ Classe 1 } & 0.8575 \\
\hline Classe 1 & 0 & 0 \\
Classe 2 & 0.8575 &
\end{tabular}

As informações agrupadas na Tabela 5 demostram que todas as amostras foram classificadas, não havendo amostras anômalas no modelo criado. As distâncias interclasses mostram um valor maior entre classes distintas do que entre os membros de uma mesma classe. O mesmo ocorre com os resíduos interclasses, pois esse parâmetro avalia o quão ajustado ao modelo criado estão aos dados. Os parâmetros avaliados por SIMCA indicam adequabilidade para predições de novas amostras por EDXRF.

Além dessas respostas referentes aos parâmetros do modelo estatístico faz-se necessário avaliar a reclassificação dos dados originais. Esse procedimento tem como intuito verificar se amostras indicadas inicialmente em uma das classes realmente pertencem a ela. A Figura 4 agrupa os resultados de forma gráfica das predições do modelo SIMCA.

Para o caso do Lado Interno (Figura 4A), observamos que a classificação inicial coincide com a classificação gerada pelo modelo SIMCA. Nesse caso, observamos ambas as respostas abaixo do eixo das amostras (eixo x). A mesma observação pode ser feita para as amostras do Lado Externo (Figura 4B), mas, nesse caso, as respostas estão acima do eixo. É possível observar que para a maioria das amostras iniciais a classificação foi realizada corretamente. Entretanto, com o intuito de avaliarmos com maior detalhamento essas respostas geradas pelo modelo SIMCA, adaptamos o modelo de tabela de contingência $2 \times 2 .{ }^{118-120}$

Essa abordagem para análise foi utilizada, pois as respostas fornecidas pelo SIMCA são qualitativas, isso é, são respostas sobre classificação correta ou errada (positiva/negativa) ao indicado inicialmente. Como essa resposta é binária (certo/errado), não se pode utilizar meios quantitativos para realizar essa análise. Logo, estabelece-se essa probabilidade por meio de quatro possíveis cenários de resposta binária. ${ }^{118}$

$\mathrm{Na}$ Tabela 6 pode-se observar a frequência (porcentagem) das amostras classificadas corretamente e incorretamente em cada uma das classes modeladas com SIMCA. Com base nesses valores, podem-se analisar os parâmetros de sensibilidade e especificidade do modelo para a predição de uma nova amostra de para-brisas aplicando a técnica EDXRF. Logo, na Tabela 6 estão reunidos os valores avaliados e na Tabela 7 os parâmetros de descrição da performance do modelo.

Os valores dos parâmetros indicam que o modelo desenvolvido apresenta sensibilidade de 0.793 , uma especificidade de 0.815 e uma eficiência de 0.804 para futuras predições utilizando a mesma técnica analítica para vidros automobilísticos (para-brisas).

Tabela 6. Tabela de contingência $2 \times 2$ adaptada ${ }^{118-120}$

\begin{tabular}{cccc}
\hline \multirow{2}{*}{ Indicação } & \multicolumn{2}{c}{ Predição } & \multirow{2}{*}{ Total (\%) } \\
\cline { 2 - 3 } & Lado Interno (\%) & Lado Externo (\%) & \\
\hline Lado Interno & $82.14(23)$ & $17.86(05)$ & $100.00(28)$ \\
Lado Externo & $21.43(06)$ & $78.57(22)$ & $100.00(28)$ \\
\hline $\begin{array}{c}\text { Total de amostras } \\
\text { analisadas }\end{array}$ & $103.57(29)$ & $96.43(27)$ & $200.00(56)$ \\
\hline
\end{tabular}

Tabela 7. Parâmetros de descrição da performance ${ }^{118-120}$

\begin{tabular}{cc}
\hline Sensibilidade & 0.793 \\
\hline $\begin{array}{c}\text { Taxa de falsos negativos }(\mathrm{FN}) \\
(1-\text { sensibilidade })\end{array}$ & 0.207 \\
\hline Especificidade & 0.815 \\
\hline $\begin{array}{c}\text { Taxas de falsos positivos (FP) } \\
(1 \text { - especificidade })\end{array}$ & 0.185 \\
\hline Eficiência & 0.804 \\
\hline
\end{tabular}

(A)

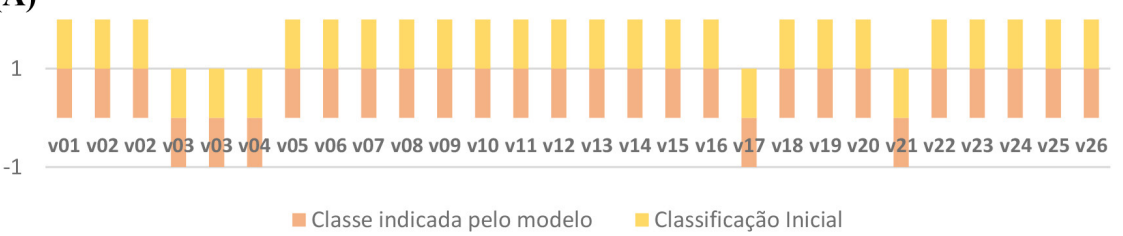

(B)

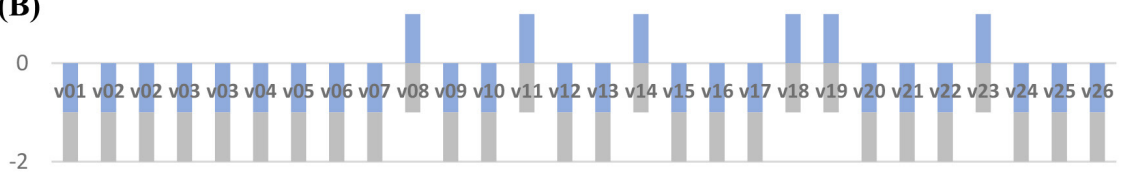

n Classe indicada pelo modelo $\quad$ Classificação Inicial

Figura 4. Classes indicadas pelo modelo SIMCA para as amostras de vidros do Lado (A) Interno e (B) Externo 
A classificação das amostras indicou uma taxa de acerto de aproximadamente $80 \%$ (Tabela 6). Todavia, é importante ressaltar que há detalhes relacionados a erros experimentais e que devem ser levados em consideração, como por exemplo: i) tempo de exposição ao feixe de raios-x; ${ }^{127}$ ii) interferência ou sobreposição de picos por outros elementos requerendo medições adicionais para realizar as distinções; ${ }^{127}$ iii) componentes presentes na linha do feixe de radiação; ${ }^{127}$ iv) o efeito de autoabsorção e/ou espessura das amostras; ${ }^{127} \mathrm{e}$ v) sensibilidade relacionada com a fonte de radiação. ${ }^{128}$

Por outro lado, a EDXRF possui algumas vantagens quando comparada a outras técnicas espectroscópicas como AAS (Atomic Absorption Spectroscopy) e ICP-AES (Inductively Coupled Plasma Atomic Emission Spectrometry) de não ser destrutiva, ser multielemental, ofertar uma resposta rápida, com um custo mais baixo quando comparado a essas técnicas ${ }^{128}$ e ser mais fácil de interpretar. ${ }^{129}$ Todas essas vantagens são de grande valia para as análises forenses.

Para avaliarmos as previsões feitas com o modelo SIMCA, utilizamos a tabela ROC. Buscamos avaliar parâmetros como a sensibilidade e especificidade. Essa avaliação assegura que o procedimento estatístico aplicado nas amostras e técnica EDXRF gerou um modelo robusto. Observamos uma sensibilidade para diferenciação de $79 \%$ e uma especificidade para diferenciar os vidros de $81 \%$.

A análise de um único vestígio não é suficiente para determinar toda a dinâmica de um fato. A obtenção e estruturação de um conjunto probatório para compor os elementos que assegurem a provável dinâmica dos fatos torna-se imprescindível. Somada a isso, a compreensão do erro do procedimento de análise de vestígios dentro do contexto jurídico pode fornecer elementos para diminuir o risco de incertezas na interpretação, permitindo uma maior segurança jurídica. ${ }^{130,131}$

Dessa forma, por mais que esses materiais tenham em sua essência uma estrutura amorfa, entender os diferentes tipos de vidros, a composição química e as normas específicas é importante para possibilitar a avaliação em um contexto legal. Dado que a avaliação forense é complexa, a análise de vidros é uma das peças que irá compor o quebra-cabeças da investigação. Deve ser, portanto, considerada como um elemento informativo de todo o processo e a metodologia científica aplicada deverá ser sólida para fornecer credibilidade e segurança jurídica ao conjunto probatório. ${ }^{132}$

\section{CONCLUSÕES}

A utilização de PCA demonstrou potencialidade para ser utilizada de forma preliminar na visualização, redimensionamento e discriminação dos dados de vidros automobilísticos (para-brisas) analisados por EDXRF. A análise SIMCA possibilitou a elaboração de um modelo classificatório adequado, uma vez que os parâmetros para viabilidade foram respeitados. A avaliação ROC demonstrou a coesão do método estatístico, o que possibilitou a compreensão da precisão da previsibilidade do modelo SMICA.

As técnicas classificatórias multivariadas podem ser aplicadas para a correta avaliação desses possíveis vestígios. Um modelo preditivo como o proposto poderia auxiliar nos casos de acidentes de trânsito, crimes contra o patrimônio e outros. Além disso, ainda sob a perspectiva forense, a aplicação de metodologias de análise ágeis aliadas à quimiometria pode auxiliar na aplicação da lei, no entendimento da dinâmica criminosa e na constatação da materialidade do delito. Salienta-se que a interpretação desses vestígios não deve ser desconectada do contexto geral da investigação. Esse conhecimento gerado deverá auxiliar no processo de tomada de decisão e aplicação da justiça. Portanto, os procedimentos estatísticos inseridos dentro das ciências forenses poderão auxiliar no fortalecimento das discussões dos vícios, erros e problemas de interpretação das análises.

\section{AGRADECIMENTOS}

Agradecemos às agências: Conselho Nacional de Desenvolvimento Científico e Tecnológico (CNPq, projeto 465450 / 2014-8), Coordenação de Aperfeiçoamento de Pessoal de Nível Superior (CAPES, Código Financeiro 001) e Universidade de São Paulo (Bolsa Ensinar com Pesquisa) pelo apoio financeiro.

\section{REFERÊNCIAS}

1. Brasil; Ministério dos Transportes Portos e Aviação Civil (MTPA); Anuário Estatístico de Segurança Rodoviária 2010-2018; Brasília, DF, 2020.

2. Brasil; Código de Trânsito Brasileiro; Brasil, 1997.

3. Velho, J. A.; Costa, K. A.; Damasceno, C. T.; Locais de Crime - Dos vestígios à dinâmica criminosa; $1^{\mathrm{a}}$. ed., Campinas, SP, 2013.

4. Bruni, A. T.; Em Pacote Anticrime: Comentários à lei n.13.964/2019; Netto, A. V. S., Bruni, A. T., Amaral, C. do P., Saad-Diniz, E., Morais, H. D., eds.; Almedina: São Paulo, 2020; pp. 121-142.

5. Velho, J. A.; da Silva, L. A. R.; do Carmo, C. F. A.; Damasceno, C. T. M.; Em Uma Introdução Às Principais Áreas Da Criminalística; Millenninum Editora: Campinas, 2017; pp. 19-32.

6. Zadora, G.; Brożek-Mucha, Z.; Mater. Chem. Phys. 2003, 81, 345.

7. Brasil; Código de Processo Penal - Decreto-Lei n 3.689/1941; Brasil, 1941.

8. Zadora, G.; Ramos, D.; Chemom. Intell. Lab. Syst. 2010, 102, 63.

9. Taki, T.; Machida, M.; Shimada, R.; IATSS Res. 2019, 43, 84.

10. Schneider, P. M.; Seo, Y.; Rittner, C.; Int. J. Legal Med. 1999, 112, 315.

11. De Wolff, T.; Aarts, L. H. J.; van den Berge, M.; Boyko, T.; van Oorschot, R. A. H.; Zuidberg, M.; Kokshoorn, B.; Aust. J. Forensic Sci. 2019, 51, S103.

12. Abang Mustaffa, D. N.; Masuri, M. G.; Dahlan, A.; Md. Isa, K. A.; Asian J. Behav. Stud. 2017, 2, 43.

13. Kim, J.-H.; Oh, W.-T.; Choi, J.-H.; Park, J.-C.; Forensic Sci. Int. 2018 , 287, 195.

14. Torres, A. N.; Margem de erro do cálculo de velocidade a partir dos vestígios em acidentes de trânsito com tacógrafo presente, Centro Universitário de Lavras, 2019.

15. Lyu, N.; Huang, G.; Wu, C.; Duan, Z.; Li, P.; Sensors 2017, 17, 482.

16. Nakao, K.; Tatara, Y.; IATSS Res. 2019, 43, 75.

17. Koelega, H. S.; Psychopharmacology (Berl) 1995, 118, 233.

18. Høiseth, G.; Tuv, S. S.; Karinen, R.; Forensic Sci. Int. 2016, $268,35$.

19. Damacena, G. N.; Malta, D. C.; Boccolini, C. S.; Souza Júnior, P. R. B. de; Almeida, W. da S. de; Ribeiro, L. S.; Szwarcwald, C. L.; Cien. Saude Colet. 2016, 21, 3777.

20. Younis, R.; Adel, R.; Mansoura J. Forensic Med. Clin. Toxicol. 2019, $27,1$.

21. Aktas, N.; Gulacti, U.; Lok, U.; Aydin, I.; Borta, T.; Celik, M.; Bull. Emerg. Trauma 2018, 6, 64.

22. Camargo Filho, W. X.; Telles, B.; Andrade, C. A.; Sercheli, M. S.; Kawano, N. M.; Soares, R. M.; Vicente, A. N.; Corrêa, R. S.; Gomes, J. A.; Rev. Bras. Crim. 2016, 5, 7.

23. Campos, K. R. M.; O Alferes 2017, 27, 203.

24. Aldegheishem, A.; Yasmeen, H.; Maryam, H.; Shah, M.; Mehmood, A.; Alrajeh, N.; Song, H.; Sensors 2018, 18, 1983.

25. Otuki, G. F.; Dissertação de Mestrado, Universidade Federal de Alfenas, Brasil, 2019.

26. Mocellin, N.; Trabalho de Conclusão de Curso, Instituto Federal de Santa Catarina, Brasil, 2017. 
27. Bucharles, L. G. E.; Tese de Doutorado, Universidade de São Paulo, Brasil, 2014

28. Mendes, R. L.; Revista Científica do ISCTAC 2017, 2, 20.

29. Mota, H. de A.; Trabalho de Conclusão de Curso, Instituto Federal de Santa Catarina, Brasil, 2017.

30. Chen, S.; Zang, M.; Wang, D.; Yoshimura, S.; Yamada, T.; Composites, Part B 2017, 122, 47.

31. Yu, G.; Zheng, Y.; Feng, B.; Liu, B.; Meng, K.; Yang, X.; Chen, H.; Xu, J.; Comput. Struct. 2017, 193, 139.

32. Lima, V. D. O.; Ricardo, Z.; Rev. Bras. Crim. 2016, 6, 66.

33. Zou, T.; Peng, X.; Wu, W.; Cai, M.; Forensic Sci. Int. 2017, 270, 200.

34. Burghardt, T. E.; Pashkevich, A.; Mosböck, H.; Case Stud. Transp. Policy 2019, 7, 862.

35. Salvador, F. A. da S.; Miranda, G. H. B. de; Costa, K. A.; In Locais de Crimes -Dos vestígios à dinâmica Criminosa; Velho, J. A., Costa, K. A., Damasceno, C. T. M., eds.; Millenninum Editora: Campinas, 2013; pp. 253-284.

36. Xie, H.; Lin, W.; In Proceedings of the 3rd International Conference on Judicial, Administrative and Humanitarian Problems of State Structures and Economic Subjects (JAHP 2018); Atlantis Press: Paris, France, 2018.

37. Grainger, M. N. C.; Manley-Harris, M.; Coulson, S.; J. Anal. At. Spectrom. 2012, 27, 1413.

38. Girard, J. E.; Criminalistics: Forensic Science and Crime; Jones and Bartlett Publishers: Boston, 2006; pp. 97-112.

39. Buscaglia, J. A.; Anal. Chim. Acta 1994, 288, 17.

40. Rushton, K. P.; Coulson, S. A.; Newton, A. W. N.; Curran, J. M.; Forensic Sci. Int. 2011, 209, 102.

41. Mistek, E.; Fikiet, M. A.; Khandasammy, S. R.; Lednev, I. K.; Anal. Chem. 2019, 91, 637.

42. Funatsuki, A.; Takaoka, M.; Shiota, K.; Kokubu, D.; Suzuki, Y.; Anal. Sci. 2016, 32, 207.

43. Krug, F. J.; Rocha, F. R. P.; Métodos de preparo de amostras para análise elementar; $1^{\circ}$ ed.; EditSBQ: São Paulo, 2016.

44. Campbell, G. P.; Curran, J. M.; Miskelly, G. M.; Coulson, S.; Yaxley, G. M.; Grunsky, E. C.; Cox, S. C.; Forensic Sci. Int. 2009, 188, 81.

45. Rodríguez, M.; Winefordner, J.; Revista de Química (PUCP) 2007, 21, 15.

46. Akerman, M.; Natureza, Estrutura e Propriedades do Vidro, 2000, pp. $1-37$.

47. Kolb, D.; Kolb, K. E.; J. Chem. Educ. 1979, 56, 604.

48. Alves, O. L.; Quim. Nova Esc. 2001, Ediçao Esp, 13.

49. Fors, C.; Ph.D Thesis, Lund University, Sweden, 2014.

50. Yamane, M.; Asahara, Y.; Glasses for Photonics; Cambridge University Press: Cambridge, 2000.

51. Godinho, K. de O.; Dissertação de Mestrado, Universidade Estadual do Norte Fluminense, Brasil, 2004.

52. Dodds, A. J.; Pollock, E. M. C.; Land, D. P.; Forensic Glass Analysis by LA-ICP-MS: Assessing the Feasibility of Correlating Windshield Composition and Supplier, 2010, disponível em https://www.ncjrs.gov/ pdffiles1/nij/grants/232134.pdf, acessado em janeiro 2021.

53. Angell, C. A.; Science (80-. ). 1995, 267, 1924.

54. Li, J.-M.; Chu, Y.-B.; Zhao, N.; Zhou, R.; Yi, R.-X.; Guo, L.-B.; Li, J.-Y.; Li, X.-Y.; Zeng, X.-Y.; Lu, Y.-F.; Chin. J. Anal. Chem. 2016, 44 1042.

55. Felisberto, C. B.; Dissertação de Mestrado, Universidade de São Paulo, Brasil, 2006

56. Devangad, P.; Tamboli, M.; Shameem, K. M. M. M. M.; Nayak, R.; Patil, A.; Unnikrishnan, V. K. K.; Santhosh, C.; Kumar, G. A. A.; Laser Phys. 2018, 28, 015703.

57. Prandel, L. V.; Melo, V. F.; Testoni, S. A.; Brinatti, A. M.; Saab, S. da C.; Dawson, L. A.; Soil Res. 2020, 58, 151.

58. Melo, V. F.; Testoni, S. A.; Dawson, L.; de Lara, A. G.; da Silva Salvador, F. A.; Sci. Justice 2019, 59, 667.
59. Pirrie, D.; Crean, D. E.; Pidduck, A. J.; Nicholls, T. M.; Awbery, R. P.; Shail, R. K.; Geol. Soc. London, Spec. Publ. 2019, SP492.

60. Caritat, P.; Simpson, T.; Woods, B.; J. Forensic Sci. 2019, 64, 1359.

61. Stern, L. A.; Webb, J. B.; Willard, D. A.; Bernhardt, C. E.; Korejwo, D. A.; Bottrell, M. C.; McMahon, G. B.; McMillan, N. J.; Schuetter, J. M.; Hietpas, J.; Geochem., Geophys., Geosyst. 2019, 20, 913.

62. Kurfürst, U. In Solid Sample Analysis; Kurfürst, U., ed.; $1^{\text {st }}$ ed.; Springer: Berlin, Heidelberg, 1998.

63. Nomura, C. S.; Silva, C. S. da; Oliveira, P. V.; Quim. Nova 2008, 31, 104.

64. Vale, M. G. R.; Oleszczuk, N.; dos Santos, W. N. L.; Appl. Spectrosc. Rev. 2006, 41, 377.

65. Zadora, G.; Neocleous, T.; Anal. Chim. Acta 2009, 642, 266.

66. Ramos, D.; Zadora, G.; Anal. Chim. Acta 2011, 705, 207.

67. Becker, P.; Neff, C.; Hess, S.; Weis, P.; Günther, D.; J. Anal. At. Spectrom. 2020.

68. Heydon, A.; Ruddell, D.; Wolf, A.; Dorn, H.; Forensic Chem. 2018, 11, 103.

69. Jisonna, L. J.; DeYoung, P. a.; Ferens, J.; Hall, C.; Lunderberg, J. M.; Mears, P.; Padilla, D.; Peaslee, G. F.; Sampson, R.; Nucl. Instruments Methods Phys. Res. Sect. B Beam Interact. with Mater. Atoms 2011, 269, 1067.

70. Civici, N.; Vataj, E.; Rom. Rep. Phys. 2013, 65, 1265.

71. Melendez-Perez, J. J.; Correa, D. N.; Hernandes, V. V.; de Morais, D. R.; de Oliveira, R. B.; de Souza, W.; Santos, J. M.; Eberlin, M. N.; Appl. Spectrosc. 2016, 70, 1910.

72. Panchuk, V.; Yaroshenko, I.; Legin, A.; Semenov, V.; Kirsanov, D.; Anal. Chim. Acta 2018, 1040, 19.

73. Grant, B.; Sauzier, G.; Lewis, S. W.; Forensic Sci. Int. 2020, 313, 110338.

74. Lavine, B. K.; Fasasi, A.; Mirjankar, N.; Sandercock, M.; Brown, S. D.; J. Chemom. 2014, 28, 385.

75. Sacchi, C. A.; J. Opt. Soc. Am. B 1991, 8, 337.

76. Pasquini, C.; Cortez, J.; Silva, L. M. C.; Gonzaga, F. B.; J. Braz. Chem. Soc. 2007, 18, 463.

77. Hahn, D. W.; Omenetto, N.; Appl. Spectrosc. 2012, 66, 347.

78. Winefordner, J. D.; Gornushkin, I. B.; Correll, T.; Gibb, E.; Smith, B. W.; Omenetto, N.; J. Anal. At. Spectrom. 2004, 19, 1061.

79. Bridge, C. M.; Powell, J.; Steele, K. L.; Sigman, M. E.; Spectrochim. Acta Part B At. Spectrosc. 2007, 62, 1419.

80. Gupta, A.; Curran, J. M.; Coulson, S.; Triggs, C. M.; Forensic Chem. 2017, 3, 36 .

81. Slater, G. F.; Environ. Forensics 2003, 4, 13.

82. Meier-Augenstein, W.; Stable Isotope Forensics; Meier-Augenstein, W., ed.; $1^{\text {st }}$ ed.; John Wiley \& Sons, Ltd: Chichester, 2010.

83. Sjåstad, K.-E.; Simonsen, S. L.; Andersen, T.; J. Anal. At. Spectrom. 2011, 26, 325 .

84. Associação Brasileira de Normas Técnicas; NBR 9491: Vidros de segurança para veículos rodoviários - Requisitos; Brasil, 2015; p. 50.

85. Associação Brasileira de Normas Técnicas; NBR 9492: Vidros de segurança - Ensaio de ruptura - Segurança contra estilhaços; Brasil, 2014; p. 5

86. Associação Brasileira de Normas Técnicas; NBR 7199: Vidros na construção civil - Projeto, execução e aplicações; Brasil, 2016; p. 57.

87. Adame, A.; Tese de Doutorado, Universidade de São Paulo, Brasil, 2019.

88. Trejos, T.; Koch, S.; Mehltretter, A.; Forensic Chem. 2020, 18, 100223.

89. Gama, E. M.; Tese de Doutorado, Universidade Federal de Minas Gerais, Brasil, 2018.

90. Nomura, C. S.; Santos-Júnior, D.; Nunes, L. C.; Guerra, M. B. B.; de Carvalho, G. G. A.; Oliveira, P. V.; Krug, F. J.; In Métodos de preparo de amostras para análise elementar; Krug, F. J.; Rocha, F. R. P., Eds.; EditSBQ: São Paulo, 2016; pp. 137-196. 
91. Kumar, R.; Sharma, V.; TrAC - Trends Anal. Chem. 2018, 105, 191.

92. Hickman, D. A.; Forensic Sci. Int. 1981, 17, 265.

93. Hickman, D. A.; Forensic Sci. Int. 1987, 33, 23.

94. Bruni, A. T.; Rodrigues, C. H. P.; Talhavini, M.; In Fundamentos de química forense: uma análise prática da química que soluciona crimes; Bruni, A. T.; Velho, J. A.; de Oliveira, M. F., Eds.; Millenium Editora: Ribeirão Preto, 2019.

95. Almirall, J.; Trejos, T.; Lambert, K.; Forensic Science International: Synergy 2020.

96. Velho, J. A.; Costa, K. A.; Damasceno, C. T. M.; Locais de Crimes -Dos vestígios à dinâmica Criminosa, Millennium Editora: Campinas, São Paulo, 2013; p. 20.

97. Ferreira, M. M. C.; Quimiometria - Conceitos, Métodos e Aplicações; $1^{\text {a }}$ ed.; Editora Unicamp: Campinas, 2015.

98. Beebe, K. R.; Pell, R. J.; Seasholtz, M. B.; Chemometrics: A Practical Guide; 1st ed.; Wiley-Interscience: New York, 1998.

99. Miller, J. N.; Miller, J. C.; Statistics and Chemometrics for Analytical Chemistry; Fifth Ed.; Prentice Hall: Harlow, Essex, 2005.

100. Lavine, B.; Workman, J. J.; Anal. Chem. 2004, 76, 3365.

101. Chemometrics, Technical Note, Description of Pirouette Algorithms, disponível em https://chemometrix.files.wordpress.com/2014/12/191214_algorithmtn.pdf, acessada em janeiro 2021.

102. Jollife, I. T.; Cadima, J.; Philos. Trans. R. Soc., A 2016, 374.

103. Andrade, D.; Pereira-Filho, E.; Konieczynski, P.; J. Braz. Chem. Soc. 2016, $28,838$.

104. Marín, J.; Serrano, N.; Ariño, C.; Díaz-Cruz, J. M.; Chemosensors 2020 , $8,46$.

105. Rodrigues, N. V. S.; Cardoso, E. M.; Andrade, M. V. O.; Donnici, C. L.; Sena, M. M.; J. Braz. Chem. Soc. 2013, 24, 507.

106. Flumignan, D. L.; Tininis, A. G.; Ferreira, F. de O.; de Oliveira, J. E.; Anal. Chim. Acta 2007, 595, 128.

107. Arroio, A.; Lima, E. F.; Honório, K. M.; da Silva, A. B. F.; Struct. Chem. 2009, 20, 577.

108. Tominaga, Y.; Chemom. Intell. Lab. Syst. 1999, 49, 105.

109. Karlsson, T.; Forensic Sci. Int. 1999, 101, 131.

110. Sabin, J. G.; Ferrão, M. F.; Furtado, J. C.; Rev. Bras. Ciências Farm. 2004, 40, 387.

111. Tracci, F.; Trabalho de Conclusão de Curso, Universidade de São Paulo, 2010
112. Zhao, S.; Dharani, L. R.; Chai, I.; Barbat, S. D.; Eng. Failure Anal. 2006, 13, 582.

113. Russelt, M. V.; Recent trends for automotive laminated glazing, Glass Performance Day (2007), disponível em www.gpd.fi, acessado em janeiro 2021.

114. Bruni, A. T.; Leite, V. B. P.; Ferreira, M. M. M. C. C.; J. Comput. Chem. 2002, 23, 222.

115. Infometrix, Pirouette ${ }^{\circledR}$, disponível em https://infometrix.com/pirouette, acessada em janeiro 2021.

116. Lavine, B. K.; In Comprehensive Chemometrics; Elsevier: Amsterdam, 2009; pp. 587-599.

117. Fernandes, D. D. de S.; Tese de Doutorado, Universidade Federal da Paraíba, Brasil, 2016.

118. López, M. I.; Callao, M. P.; Ruisánchez, I.; Anal. Chim. Acta 2015, 891, 62.

119. Youden, W. J.; Cancer 1950, 3, 32.

120. Santana, F.; Souza, A.; Almeida, M.; Breitkreitz, M.; Filgueiras, P.; Sena, M.; Poppi, R.; Quim. Nova 2020, 43, 371.

121. Fernades, J. A.; Mugabe, D. A.; Correia, P. F.; Acta Sci. - Rev. Ensino Ciências e Matemática 2012, 14, 374.

122. Rodríguez, N. C.; Tese de Doutorado, Universidade Estatual Paulista "Júlio de Mesquita Filho", Brasil, 2020.

123. Nachtigall, F. M.; Pereira, A.; Trofymchuk, O. S.; Santos, L. S.; Nat. Biotechnol. 2020, 38, 1168.

124. Ferreira, M. M. C.; Montanari, C. A.; Gaudio, A. C.; Quim. Nova 2002, $25,439$.

125. Conselho Regional de Química - IV Região; Vidro, disponível em https://www.crq4.org.br/vidroquimicaviva, acessada em janeiro 2021.

126. Arbab, M.; Smith, D. G.; Shelestak, L. J.; Strzelecki, M. T.; CA2516539A1 2006.

127. Feng, X.; Zhang, H.; Yu, P.; Crit. Rev. Food Sci. Nutr. 2020, 1.

128. Rani, A.; Kumar, R.; Journal of Forensic Sciences \& Criminal Investigation 2019, 11, 001.

129. Palmer, P. T.; J. Chem. Educ. 2011, 88, 868.

130. Morelato, M.; Beavis, A.; Tahtouh, M.; Ribaux, O.; Kirkbride, P.; Roux, C.; Forensic Sci. Int. 2013, 226.

131. Ribaux, O.; Baylon, A.; Roux, C.; Delémont, O.; Lock, E.; Zingg, C.; Margot, P.; Forensic Sci. Int. 2010, 195, 10.

132. Ribaux, O.; Crispino, F.; Roux, C.; Aust. J. Forensic Sci. 2014, 0618, 1. 